\title{
Satellite Phase Bias Estimation with Global Networks and High-Dimensional Integer Ambiguity Fixing
}

\author{
Patrick Henkel ${ }^{*, * *}$, Dimitrios Psychas* and Christoph Günther ${ }^{*, * *, * *}$ \\ ${ }^{*}$ Technische Universität München (TUM), Munich, Germany \\ **Advanced Navigation Solutions - ANavS, Munich, Germany \\ ${ }^{* * *}$ German Aerospace Center (DLR), Oberpfaffenhofen, Germany
}

\section{BIOGRAPHIES}

Patrick Henkel received his Bachelor, Master and PhD degrees from the Technische Universität München, Munich, Germany. In 2010, he graduated with a $\mathrm{PhD}$ thesis on reliable carrier phase positioning (summa cum laude) and is now working towards his habilitation in the field of precise point positioning. He visited the Mathematical Geodesy and Positioning group at TU Delft in 2007, and the GPS Lab at Stanford University in 2008 and 2010. Dr. Henkel received the Pierre Contensou Gold Medal in 2007, the 1st prize in Bavaria at the European Satellite Navigation Competition in 2010, and the Vodafone Award for his dissertation in 2011. He is one of the founders and currently also the managing director of Advanced Navigation Solutions ANavS.

Dimitrios Psychas studied Geodesy at the National Technical University of Athens, Athens, Greece, and Earth oriented Space Science and Technology (ESPACE) at the Technische Universität München, Munich, Germany. He completed his master studies with highest distinction and a thesis on the"Estimation of GNSS satellite phase biases using a global network of reference stations". Currently, he is a PhD researcher at Fugro Intersite B.V. and TU Delft working on "Fast and reliable multi-GNSS PPP with integer ambiguity resolution", under the Horizon 2020 Marie Sklodowska-Curie TREASURE project.

Christoph Günther studied theoretical physics at the Swiss Federal Institute of Technology in Zurich. He received his diploma in 1979 and completed his $\mathrm{PhD}$ in 1984. He worked on communication and information theory at Brown Boveri and Ascom Tech. From 1995, he led the development of mobile phones for GSM and later dual mode GSM/Satellite phones at Ascom. In 1999, he became head of the research department of Ericsson in Nuremberg. Since 2003, he is the director of the Institute of Communication and Navigation at the German Aerospace Center (DLR) and since December 2004, he additionally holds a Chair at the Technische Universität München (TUM). His research interests are in satellite navigation, communication and signal processing.

\begin{abstract}
Precise point positioning requires precise knowledge of satellite phase biases, satellite position and satellite clock corrections. In this paper, a method for the estimation of these parameters with a global network of multi-frequency reference stations is presented. It includes a clustering of the reference stations. First, individual satellite phase biases, position and clock corrections are derived for each cluster. Subsequently, the solutions of each cluster are combined. We exploit the integer property of the carrier phase ambiguities and perform an integer decorrelation and fixing within each cluster and also in the multi-cluster combination. The performance of the proposed method is analyzed with Galileo measurements on both E1 and E5a of the IGS stations. We defined 16 clusters and obtained satellite phase biases with an accuracy of better than $2 \mathrm{~cm}$.
\end{abstract}

\section{INTRODUCTION}

Precise Point Positioning is becoming attractive as the user does not need any raw measurements from a reference station. Zumberge et al. have introduced Precise Point Positioning (PPP) in [1] using precise orbits and clocks obtained from a large 
network of reference stations. Kouba and Héroux describe PPP in [2] including a precise modeling of satellite antenna offsets, phase wind-up corrections, solid earth tides, ocean loading and earth rotation parameters. They have also assessed the performance using IGS (International GNSS Service) products. Precise satellite orbit and clock information can be obtained by the Bernese software as described by Dach et al. in [3].

Precise knowledge of satellite phase biases is required for PPP with integer ambiguity fixing. Ge et al. [4] used the Melbourne Wübbena combination and the ionosphere-free phase combination. These combinations were applied to satellite-satellite single difference measurements of 450 IGS stations to estimate the single-difference widelane biases and phase biases. The estimation is performed in two steps, i.e. a first step for the widelane ambiguity fixing, and a subsequent step for fixing the narrowlane ambiguities. The estimated phase biases of [4] vary by only 0.4 cycles per day for some GPS satellites. Ge et al. also show that their corrections enable a narrowlane (NL) integer ambiguity fixing, i.e. $91 \%$ of the float NL ambiguity estimates are deviating by at most 0.1 cycles from the nearest integer.

Laurichesse et al. [5] estimated undifferenced satellite phase clocks, station clocks, satellite differential code/ phase bias, station differential code/ phase biases, station coordinate corrections, satellite orbit corrections and ambiguities in a Kalman filter. They used the same combinations as Ge et al. [4], but processed undifferenced measurements. The assumptions on the process noise statistics are accurately described for each state parameter. Laurichesse et al. [5] analyzed the evolution of the narrowlane pseudorange minus phase biases over the whole year 2008 for all GPS satellites. They distinguished between blocs IIA and IIR satellites and observed a drift of only 3 narrowlane cycles per year.

Wen et al. [6] proposed to estimate the undifferenced satellite phase biases, non-dispersive geometry correction terms including their time derivatives, slant ionospheric delays, and carrier phase ambiguities in a Kalman filter. The ambiguity fixing was triggered based on the stability of the float solution and the statistics of the Kalman filter. The method was applied to the regional network of SAPOS reference stations in Germany.

Carrier phase integer ambiguity fixing is essential for precise satellite phase bias estimates. Blewitt proposed a sequential ambiguity fixing in [8], which partially exploits the correlation between float ambiguities. The correlation was obtained from a triangular decomposition of the float ambiguity covariance matrix. Teunissen developed the famous Least-squares AMBiguity Decorrelation Adjustment (LAMBDA) method in [7] to solve the integer least-squares problem. The LAMBDA method includes an integer decorrelation and a sequential tree search to find the integer ambiguities which minimize the sum of squared ambiguity residuals. Teunissen provides an expression for the success rate of integer bootstrapping based on the cumulative Gaussian distribution in [9].

Brack et al. [10] proposed a sequential Best-Integer Equivariant (BIE) estimator for high-dimensional integer ambiguity fixing. The authors performed $n$ one-dimensional searches instead of one $n$-dimensional search, which is much more efficient. The Sequential BIE was used for satellite phase bias estimation with 20 reference stations.

Henkel et al. [11] developed an ambiguity transformation for GLONASS double difference carrier phase measurements to enable integer ambiguity fixing for FDMA-moduated signals. The transformation was used for joint ambiguity fixing of GPS and GLONASS.

In this paper, a method for undifferenced satellite phase bias estimation with a large global network of reference stations is presented. The method outperforms previous methods by

- splitting the global network into several clusters and by introducing a parameter mapping, which maximizes the number of integer-valued parameters that can be estimated without rank defect on a global perspective

- fixing all integer-valued ambiguities within each cluster using integer decorrelation

- fixing the between cluster differential ambiguities using integer decorrelation

The method consists of two steps as shown in Fig. 1. The first step includes the splitting of the global network into several clusters and a separate processing of undifferenced and uncombined measurements of each cluster. The second step includes the combination of the individual solutions.

We perform a clustering of the global receiver network for the following reasons:

- selection of common reference satellite and common reference receiver for all measurements only feasible with regional coverage

- common visibility at reference receiver and any other receiver within a cluster enables relationship between real-valued undifferenced ambiguity/ phase biases using double difference integer ambiguities

- reduced dimensions of measurements and states within each cluster enables integer ambiguity decorrelation and fixing

- selection of receiver-independent reference satellite enables transformation instead of re-estimation of clock offsets, receiver phase biases and double difference integer ambiguities in case of changing reference satellite 
Cluster 1

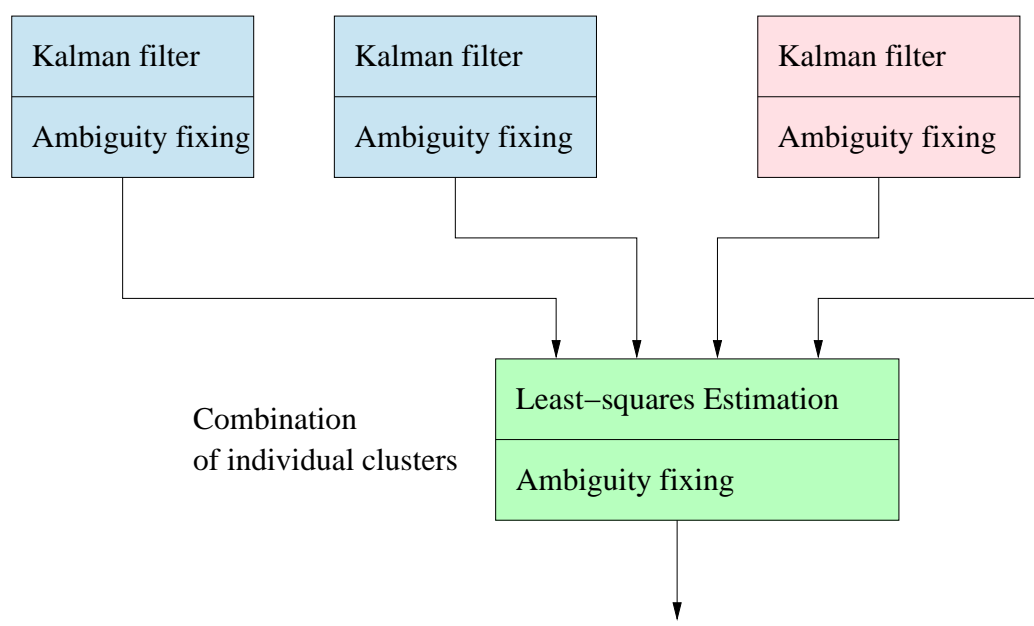

orbital, clock and phase bias corrections of satellites
Cluster C

Kalman filter

Ambiguity fixing

Fig. 1: Functional diagram for satellite phase bias estimation with clustering of global network

The optimum size of the cluster depends on the geographical distribution of the reference stations. A large cluster size provides more measurements related to a certain satellite, which improves the accuracy of the respective clock and phase bias estimates. However, there are also two arguments for small to moderate cluster sizes:

- integer ambiguity decorrelation only feasible with small to moderate cluster size

- joint visibility between reference receiver and any other receiver of cluster reduces with size of cluster

Thus, the optimum cluster size is a trade-off between maximizing the number of measurements and maximizing common satellite visibility.

Fig. 2 shows a map with the locations of the 491 IGS stations. The stations are grouped into 16 clusters with the reference cluster being in Europe. Each cluster has a reference receiver, being denoted by a circle. It is selected based on the distances of the receivers' positions from the mean value of all station coordinates within one cluster.

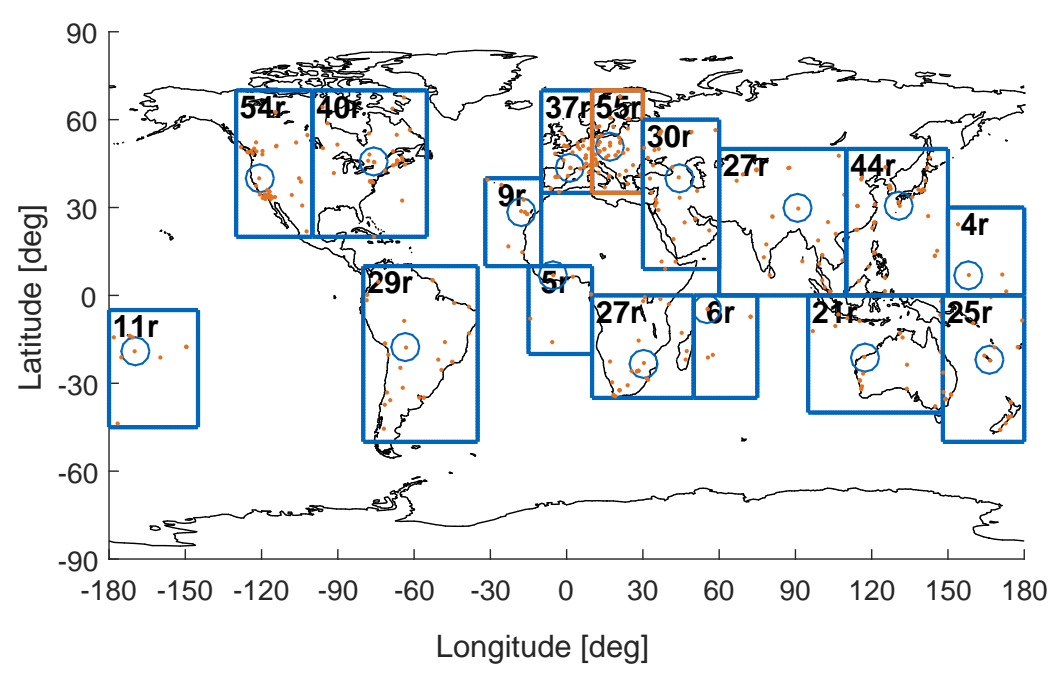

Fig. 2: Map of IGS stations with 16 clusters. The number of receivers per cluster is provided in the upper left corner of each cluster. The reference receiver of each cluster is additionally highlighted. 


\section{MEASUREMENT MODEL}

The undifferenced carrier phase measured at receiver $r \in\{1, \ldots, R\}$ on frequency $m=\{1, \ldots, M\}$ of satellite $k=$ $\{1, \ldots, K\}$ is modeled as

$$
\lambda_{m} \varphi_{r, m}^{k}=\vec{e}_{r}^{k}\left(\vec{x}_{r}-\vec{x}^{k}\right)+c\left(\delta \tau_{r}-\delta \tau^{k}\right)+m_{\mathrm{T}}\left(E_{r}^{k}\right) T_{\mathrm{z}, r}-q_{1 m}^{2} I_{r, 1}^{k}+\lambda_{m} N_{r, m}^{k}+\beta_{r, m}-\beta_{m}^{k}+\varepsilon_{r, m}^{k} \quad \forall r, m, k,
$$

with the wavelength $\lambda_{m}$, the carrier phase measurement $\varphi_{r, m}^{k}$ in units of cycles, the line of sight vector $\vec{e}_{r}^{k}$ pointing from the satellite to the receiver, the positions $\vec{x}_{r}$ and $\vec{x}^{k}$ of the receiver and satellite, the speed of light $c$, the clock offsets $\delta \tau_{r}$ and $\delta \tau^{k}$ of the receiver and satellite, the tropospheric mapping function $m_{\mathrm{T}}$ depending on the elevation angle $E_{r}^{k}$, the tropospheric zenith delay $T_{\mathrm{z}, r}$, the ratio of frequencies $q_{1 m}=f_{1} / f_{m}$, the ionospheric slant delay $I_{r, 1}^{k}$ on $f_{1}$, the integer ambiguity $N_{r, m}^{k}$, the receiver phase bias $\beta_{r, m}$, the satellite phase bias $\beta_{m}^{k}$ and the phase noise $\varepsilon_{r, m}^{k}$.

The pseudoranges are modeled similarly as

$$
\rho_{r, m}^{k}=\vec{e}_{r}^{k}\left(\vec{x}_{r}-\vec{x}^{k}\right)+c\left(\delta \tau_{r}-\delta \tau^{k}\right)+m_{\mathrm{T}}\left(E_{r}^{k}\right) T_{\mathrm{z}, r}+q_{1 m}^{2} I_{r, 1}^{k}+b_{r, m}-b_{m}^{k}+\Delta \rho_{\mathrm{MP}_{r, m}}^{k}+\eta_{r, m}^{k} \quad \forall r, m, k,
$$

with the code biases $b_{r, m}$ and $b_{m}^{k}$, the pseudorange multipath $\Delta \rho_{\mathrm{MP}_{r, m}}^{k}$ and the pseudorange noise $\eta_{r, m}^{k}$.

\section{PARAMETER MAPPING}

In this section, we introduce a parameter mapping that (a) combines some of the above parameters to enable a full-rank system of equation and that (b) combines ambiguities mainly with ambiguities to preserve the integer property of ambiguities. The mapping requires the selection of a reference receiver and reference satellite being denoted by the lower/ upper index ref. As the reference receiver and reference satellite are cluster dependent, we introduce the index $c=\{1, \ldots, C\}$ to denote all cluster dependent parameters.

The rank-defect of the absolute clock offset estimation is prevented by mapping the satellite clock offset of the reference satellite ref of cluster $c$ to the receiver clock offset, i.e.

$$
\delta \tilde{\tau}_{r, c}:=\delta \tau_{r}-\delta \tau^{\mathrm{ref}, c} \quad \forall r, c .
$$

The clock offsets of the other satellites are adjusted respectively:

$$
\delta \tilde{\tau}^{k, c}:=\delta \tau^{k}-\delta \tau^{\mathrm{ref}, c} \quad \forall k, c .
$$

The code biases $b_{r, m}$ and $b_{m}^{k}$ of the first two frequencies can be mapped to the clock offset and ionospheric delay:

$$
\left(\begin{array}{c}
b_{r, 1}-b_{1}^{k} \\
b_{r, 2}-b_{2}^{k} \\
b_{r, 3}-b_{3}^{k} \\
\vdots \\
b_{r, M}-b_{M}^{k}
\end{array}\right)=\Lambda\left(\begin{array}{c}
b_{\delta \tau_{r}}-b_{\delta \tau^{k}} \\
b_{I_{r}^{k}} \\
\tilde{b}_{r, 3}-\tilde{b}_{3}^{k} \\
\vdots \\
\tilde{b}_{r, M}-\tilde{b}_{M}^{k}
\end{array}\right)
$$

with

$$
\Lambda=\left(\begin{array}{ccccc}
1 & 1 & 0 & \cdots & 0 \\
1 & q_{12}^{2} & 0 & \cdots & 0 \\
1 & q_{13}^{2} & 1 & & 0 \\
\vdots & \vdots & & \ddots & \\
1 & q_{1 M}^{2} & 0 & & 1
\end{array}\right)
$$

Solving for the biases of the clock offsets and ionospheric delays gives:

$$
\left(\begin{array}{c}
b_{\delta \tau_{r}}-b_{\delta \tau^{k}} \\
b_{I_{r}^{k}} \\
\tilde{b}_{r, 3}-\tilde{b}_{3}^{k} \\
\vdots \\
\tilde{b}_{r, M}-\tilde{b}_{M}^{k}
\end{array}\right)=\Lambda^{-1} \cdot\left(\begin{array}{c}
b_{r, 1}-b_{1}^{k} \\
b_{r, 2}-b_{2}^{k} \\
b_{r, 3}-b_{3}^{k} \\
\vdots \\
b_{r, M}-b_{M}^{k}
\end{array}\right)
$$


The receiver clock offset is extended to:

$$
\delta \tilde{\tau}_{r, c}:=\delta \tau_{r}-\delta \tau^{\mathrm{ref}, c}+\sum_{m=1}^{M} \gamma_{1 m} \cdot\left(b_{r, m}-b_{m}^{\mathrm{ref}, c}\right)
$$

with $\gamma_{1 m}$ being the element of the 1 -st row and $m$-th column of $\Lambda^{-1}$. The satellite clock offsets are adjusted respectively, i.e.

$$
\delta \tilde{\tau}^{k, c}:=\delta \tau^{k}-\delta \tau^{\mathrm{ref}, c}+\sum_{m=1}^{M} \gamma_{1 m} \cdot\left(b_{m}^{k}-b_{m}^{\mathrm{ref}, c}\right) .
$$

The slant ionospheric delay is adjusted similar as:

$$
\tilde{I}_{r}^{k}:=I_{r}^{k}+\sum_{m=1}^{M} \gamma_{2 m} \cdot\left(b_{r, m}-b_{m}^{k}\right)
$$

with $\gamma_{2 m}$ denoting the element of the 2 -nd row and $m$-th column of $\Lambda^{-1}$.

The estimation of an individual receiver phase bias for each receiver, of an individual satellite phase bias for each satellite, and of an individual integer ambiguity for each link is not feasible due to a rank deficient. We perform the following parameter mappings to overcome the rank defect:

- mapping of phase bias of reference receiver to satellite phase biases

- mapping of ambiguities of reference receiver to satellite phase biases

- mapping of ambiguities of reference satellite to receiver phase biases

Additionally, the phase biases have to be corrected for the code biases being mapped into the clock offsets and ionospheric delay. Thus, the receiver phase bias with lumped code biases and ambiguities of reference receiver and satellite is given by

$$
\begin{aligned}
\tilde{\beta}_{r, c, m}:= & \beta_{r, m}-\beta_{\text {ref }, c, m} \\
& -\sum_{m=1}^{M} \gamma_{1 m} \cdot\left(b_{r, m}-b_{\text {ref }, c, m}\right)+\sum_{m=1}^{M} q_{1 m}^{2} \cdot \gamma_{2 m} \cdot\left(b_{r, m}-b_{\text {ref }, c, m}\right) \\
& +N_{r, m}^{\text {ref }, c}-N_{\text {ref }, c, m}^{\text {ref },} \quad \forall r, c, m .
\end{aligned}
$$

The satellite phase bias is adjusted respectively, i.e.

$$
\begin{aligned}
\tilde{\beta}_{m}^{k, c}:= & \beta_{m}^{k}-\beta_{\text {ref }, c, m} \\
& -\sum_{m=1}^{M} \gamma_{1 m} \cdot\left(b_{m}^{k}-b_{\text {ref }, c, m}\right)+\sum_{m=1}^{M} q_{1 m}^{2} \cdot \gamma_{2 m} \cdot\left(b_{m}^{k}-b_{\text {ref }, c, m}\right) \\
& -N_{\text {ref }, c, m}^{k} \quad \forall k, c, m .
\end{aligned}
$$

The individual integer ambiguity is related to the integer ambiguity of the reference receiver and reference satellite, which results in the well-known double difference integer ambiguity:

$$
\tilde{N}_{r, c, m}^{s_{r}^{\bar{k}}}:=\left(N_{r, m}^{s_{r}^{\bar{k}}}-N_{r, m}^{\mathrm{ref}, c}\right)-\left(N_{\mathrm{ref}, c, m}^{s_{r}^{\bar{k}}}-N_{\text {ref, }, c, m}^{\mathrm{ref}, c}\right) \quad \forall r, c, \bar{k}
$$

where $s_{r}$ denotes the subset of visible satellites at the $r$-th receiver and $\bar{k}$ is the satellite index within this subset.

The undifferenced carrier phase measurements of Equation (1) can be expressed in terms of the reduced parameter set $\delta \tilde{\tau}_{r, c}$, $\delta \tilde{\tau}^{k, c}, \tilde{\beta}_{r, c, m}, \tilde{\beta}_{m}^{k, c}$ and $\tilde{N}_{r, c, m}^{k}$. Some parameters vanish and do not have to be estimated for $r=\operatorname{ref}$ or $s_{r}^{\bar{k}}=$ ref as shown in Equations (13) and (14). 


$$
\begin{aligned}
& \lambda_{m} \varphi_{r, m}^{k}=\vec{e}_{r}^{k}\left(\vec{x}_{r}-\vec{x}^{k}\right)+m_{\mathrm{T}}\left(E_{r}^{k}\right) T_{\mathrm{z}, r}-q_{1 m}^{2} \tilde{I}_{r, 1}^{k} \\
& +\left\{\begin{array}{lllll}
c \delta \tilde{\tau}_{r, c}-c \delta \tilde{\tau}^{k, c} & +\tilde{\beta}_{r, c, m} & -\tilde{\beta}_{m}^{k, c}+\lambda_{m} \tilde{N}_{r, c, m}^{k} & +\varepsilon_{r, m}^{k} & r \neq \text { ref, } k \neq \text { ref } \\
c \delta \tilde{\tau}_{r, c} & +\tilde{\beta}_{r, c, m} & -\tilde{\beta}_{m, c}^{k, c} & +\varepsilon_{r, m}^{k} & r \neq \text { ref, } k=\text { ref } \\
c \delta \tilde{\tau}_{r, c}-c \delta \tilde{\tau}^{k, c} & & -\tilde{\beta}_{m, c}^{k, c} & +\varepsilon_{r, m}^{k} & r=\text { ref, } k \neq \text { ref } \\
c \delta \tilde{\tau}_{r, c} & & -\tilde{\beta}_{m}^{k, c} & +\varepsilon_{r, m}^{k} & r=\text { ref, } k=\text { ref. }
\end{array}\right. \\
& \rho_{r, m}^{k}=\vec{e}_{r}^{k}\left(\vec{x}_{r}-\vec{x}^{k}\right)+m_{\mathrm{T}}\left(E_{r}^{k}\right) T_{\mathrm{z}, r}+q_{1 m}^{2} \tilde{I}_{r, 1}^{k} \\
& +\left\{\begin{array}{llll}
c \delta \tilde{\tau}_{r, c}-c \delta \tilde{\tau}^{k, c} & +\Delta \rho_{\mathrm{MP}_{r, m}}^{k} & +\eta_{r, m}^{k} & r \neq \text { ref, } k \neq \text { ref } \\
c \delta \tilde{\tau}_{r, c} & +\Delta \rho_{\mathrm{MP}_{r, m}} & +\eta_{r, m}^{k} & r \neq \text { ref, } k=\text { ref } \\
c \delta \tilde{\tau}_{r, c}-c \delta \tilde{\tau}^{k, c} & +\Delta \rho_{\mathrm{MP}_{r, m}}^{k} & +\eta_{r, m}^{k} & r=\text { ref, } k \neq \text { ref } \\
c \delta \tilde{\tau}_{r, c} & +\Delta \rho_{\mathrm{MP}_{r, m}} & +\eta_{r, m}^{k} & r=\text { ref, } k=\text { ref. }
\end{array}\right.
\end{aligned}
$$

\section{SINGLE CLUSTER SOLUTIONS WITH KALMAN FILTER}

In this section, we briefly describe the estimation of satellite positions, clock offsets and phase biases for one individual cluster.

The carrier phase and pseudorange measurements of all receivers $r \in\{1, \ldots, R\}$ and frequencies $m \in\{1, \ldots, M\}$ in cluster $c$ of a certain epoch are stacked in a column vector, i.e.

$$
\begin{aligned}
z= & \left(\lambda_{1} \varphi_{1,1}^{1}, \ldots, \lambda_{1} \varphi_{1,1}^{K}, \ldots, \lambda_{1} \varphi_{R, 1}^{1}, \ldots, \lambda_{1} \varphi_{R, 1}^{K}, \ldots,\right. \\
& \lambda_{M} \varphi_{1, M}^{1}, \ldots, \lambda_{M} \varphi_{1, M}^{K}, \ldots, \lambda_{M} \varphi_{R, M}^{1}, \ldots, \lambda_{M} \varphi_{R, M}^{K}, \\
& \rho_{1,1}^{1}, \ldots, \rho_{1,1}^{K}, \ldots, \rho_{R, 1}^{1}, \ldots, \rho_{R, 1}^{K}, \ldots, \\
& \left.\rho_{1, M}^{1}, \ldots, \rho_{1, M}^{K}, \ldots, \rho_{R, M}^{1}, \ldots, \rho_{R, M}^{K}\right)^{\mathrm{T}} .
\end{aligned}
$$

Similarly, all unknowns of cluster $c$ are stacked in the state vector $x$ given by

$$
\begin{aligned}
x=( & \left(\vec{x}^{1}\right)^{\mathrm{T}}, \ldots,\left(\vec{x}^{K}\right)^{\mathrm{T}}, \\
& c \delta \tilde{\tau}_{1, c}, \ldots, c \delta \tilde{\tau}_{R, c}, \delta \tilde{\tau}^{1, c}, \ldots, \delta \tilde{\tau}^{K, c}, \\
& T_{z, 1}, \ldots, T_{z, R}, \\
& \tilde{I}_{1,1}^{1}, \ldots, \tilde{I}_{1,1}^{K}, \ldots, \tilde{I}_{R, 1}^{1}, \ldots, \tilde{I}_{R, 1}^{K}, \\
& \tilde{\beta}_{1, c, 1}, \ldots, \tilde{\beta}_{R, c, 1}, \ldots, \tilde{\beta}_{1, c, M}, \ldots, \tilde{\beta}_{R, c, M}, \\
& \tilde{\beta}_{1}^{1, c}, \ldots, \tilde{\beta}_{1}^{K, c}, \ldots, \tilde{\beta}_{M}^{1, c}, \ldots, \tilde{\beta}_{M}^{K, c}, \\
& \tilde{N}_{1, c, 1}^{1}, \ldots, \tilde{N}_{1, c, 1}^{K}, \ldots, \tilde{N}_{R, c, 1}^{1}, \ldots, \tilde{N}_{R, c, 1}^{K}, \ldots \\
& \left.\tilde{N}_{1, c, M}^{1}, \ldots, \tilde{N}_{1, c, M}^{K}, \ldots, \tilde{N}_{R, c, M}^{1}, \ldots, \tilde{N}_{R, c, M}^{K}\right)^{\mathrm{T}} .
\end{aligned}
$$

As the satellite positions and clock offsets are known from the broadcast orbits with an accuracy of $1 \mathrm{~m}$ and as the order of magnitude of atmospheric errors and satellite phase biases is also known, we introduce some prior knowledge on these state parameters:

$$
\begin{aligned}
\bar{x}= & \left(\left(\overrightarrow{\bar{x}}^{1}\right)^{\mathrm{T}}, \ldots,\left(\overrightarrow{\bar{x}}^{K}\right)^{\mathrm{T}},\right. \\
& \delta \tilde{\bar{\tau}}^{1, c}, \ldots, \delta \tilde{\bar{\tau}}^{K, c} \\
& \bar{T}_{z, 1}, \ldots, \overline{\bar{T}}_{z, R}, \\
& \tilde{\bar{I}}_{1,1}^{1}, \ldots, \tilde{\bar{I}}_{1,1}^{K}, \ldots, \tilde{\bar{I}}_{R, 1}^{1}, \ldots, \tilde{\bar{I}}_{R, 1}^{K}, \\
& \tilde{\widetilde{\beta}}_{1, c, 1}, \ldots, \tilde{\bar{\beta}}_{R, c, 1}, \ldots, \tilde{\bar{\beta}}_{1, c, M}, \ldots, \tilde{\bar{\beta}}_{R, c, M}, \\
& \left.\tilde{\bar{\beta}}_{1}^{1, c}, \ldots, \tilde{\bar{\beta}}_{1}^{K, c}, \ldots, \tilde{\tilde{\beta}}_{M}^{1, c}, \ldots, \tilde{\bar{\beta}}_{M}^{K, c}\right)^{\mathrm{T}},
\end{aligned}
$$


with a priori known covariance matrix $\Sigma_{\bar{x}}$.

Both measurements and prior information are linear dependent on the state vector. Thus, $z$ and $\bar{x}$ of epoch $n$ are combined to

$$
\left(\begin{array}{c}
z_{n} \\
\bar{x}_{n}
\end{array}\right)=H_{n} x_{n}+\left(\begin{array}{c}
\eta_{z, n} \\
\eta_{\bar{x}, n}
\end{array}\right)
$$

with $H_{n}$ being the projection of the state vector into the measurements at epoch $n$. We use a standard Kalman filter to estimate $x_{n}$ from the extended measurement vector, i.e. the a posteriori state estimate $\hat{x}_{n}^{+}$at epoch $n$ is given by

$$
\hat{x}_{n}^{+}=\hat{x}_{n}^{-}+K_{n}\left(z_{n}-H_{n} \hat{x}_{n}^{-}\right),
$$

with the Kalman gain $K_{n}$.

The estimated state vector also includes

$$
M \cdot \sum_{r=1, r \neq \mathrm{ref}}^{R}\left(K_{r}-1\right)
$$

double difference ambiguities. A typical cluster with $R=40$ dual-frequency receivers and an average of 9 visible satellites per receiver results in $2 \cdot(40-1) \cdot(9-1)=624$ double difference ambiguities. The fixing of a few hundred of ambiguities to integers is computationally demanding. The integer transformation $Z$ of Teunissen [7] is still feasible to decorrelate the float ambiguity estimates, i.e.

$$
\hat{N}^{\prime}=Z \hat{\tilde{N}}
$$

where $\hat{\tilde{N}}$ represents the ambiguity estimates of all receivers and frequencies in cluster $c$ as obtained from the Kalman filter:

$$
\begin{aligned}
\hat{\tilde{N}}= & \left(\hat{\tilde{N}}_{1, c, 1}^{1}, \ldots, \hat{\tilde{N}}_{1, c, 1}^{K}, \ldots, \hat{\tilde{N}}_{R, c, 1}^{1}, \ldots, \hat{\tilde{N}}_{R, c, 1}^{K}, \ldots,\right. \\
& \left.\hat{\tilde{N}}_{1, c, M}^{1}, \ldots, \hat{\tilde{N}}_{1, c, M}^{K}, \ldots, \hat{\tilde{N}}_{R, c, M}^{1}, \ldots, \hat{\tilde{N}}_{R, c, M}^{K}\right)^{\mathrm{T}} .
\end{aligned}
$$

The subsequent ambiguity fixing can not be performed by a tree search due to computational limitations. However, a sequential adjustment as described by Blewitt in [8] is still feasible. The $i$-th ambiguity conditioned on all previous ones is given by:

$$
\hat{N}_{i \mid 1, \ldots, i-1}^{\prime}=\hat{N}_{i}^{\prime}-\sum_{j=1}^{i-1} \gamma_{i j}\left(\hat{N}_{j \mid 1, \ldots, j-1}^{\prime}-\left[\hat{N}_{j \mid 1, \ldots, j-1}^{\prime}\right]\right)
$$

with

$$
\gamma_{i j}=\frac{\sigma_{\hat{N}_{i}^{\prime} \hat{N}_{j \mid 1, \ldots, j-1}^{\prime}}}{\sigma_{\hat{N}_{j \mid 1, \ldots, j-1}^{\prime}}^{2}} .
$$

The conditioned decorrelated ambiguities are rounded to their nearest integer number:

$$
\check{N}_{i}^{\prime}=\left[\hat{N}_{i \mid 1, \ldots, i-1}^{\prime}\right] \text {. }
$$

The real-valued parameters of Eq. (16) are adjusted after the ambiguity fixing to improve their accuracy.

\section{COMBINATION OF CLUSTERS}

This section describes the combination of the satellite positions, clock offsets and phase bias estimates of all clusters. We derive a multi-cluster solution to achieve the following benefits:

- satellites being visible from more than one cluster provide multiple correlated satellite position, clock and phase bias estimates

- selection of a reference cluster $c_{\text {ref }}$ enables relation of cluster-dependent satellite phase biases to satellite phase biases of reference cluster and to exploit integer property of double difference ambiguities related to reference satellite of reference cluster and any other satellite being jointly visible at reference cluster and any other cluster, and being observed by the reference stations of both clusters. 
The satellite position estimate of the $c$-th cluster can be related to the true satellite position:

$$
\hat{\vec{x}}^{k, c}=\hat{\vec{x}}^{k}+\eta_{\hat{\vec{x}}^{k, c}},
$$

where the true position is cluster-independent and $\eta_{\hat{\vec{x}}^{k, c}}$ denotes the error of the estimate.

The estimates of the cluster-dependent satellite clock offsets of Equation (3) are related to the satellite clock offsets of the reference cluster and differential (cluster to reference cluster) satellite clock offsets, i.e.

$$
\begin{aligned}
\delta \hat{\tilde{\tau}}^{k, c} & :=\delta \tau^{k}-\delta \tau^{\mathrm{ref}, c}+\eta_{\delta \hat{\tilde{\tau}}^{k, c}} \\
& =\delta \tau^{k}-\delta \tau^{\mathrm{ref}, c_{\mathrm{ref}}}-\left(\delta \tau^{\mathrm{ref}, c}-\delta \tau^{\mathrm{ref}, c_{\mathrm{ref}}}\right)+\eta_{\delta \hat{\tilde{\tau}}^{k, c}}
\end{aligned}
$$

The estimates of the satellite phase biases $\tilde{\beta}_{m}^{k, c}$ of Equation (11) include satellite and/ or constellation-dependent parameters. Therefore, we rewrite the satellite phase bias estimates as

$$
\hat{\tilde{\beta}}_{m}^{k, c}=u(k, m)+v(c, m)+w(k, c, m)+\eta_{\tilde{\beta}_{m}^{k, c}}
$$

with

$$
\begin{aligned}
u(k, m) & =\beta_{m}^{k}-\sum_{m=1}^{M}\left(\gamma_{1 m}-q_{1 m}^{2} \gamma_{2 m}\right) b_{m}^{k} \\
v(c, m) & =-\beta_{\text {ref }, c, m}+\sum_{m=1}^{M}\left(\gamma_{1 m}-q_{1 m}^{2} \gamma_{2 m}\right) b_{\text {ref }, c, m} \\
w(k, c, m) & =-N_{\text {ref }, c, m}^{k} .
\end{aligned}
$$

The satellite phase bias estimates of any cluster $c$ can be related to the satellite phase bias estimates of the reference cluster, i.e.

$$
\begin{aligned}
\hat{\tilde{\beta}}_{m}^{k, c}= & u(k, m)+v\left(c_{\mathrm{ref}}, m\right)+w\left(k, c_{\mathrm{ref}}, m\right) \\
& +\left(v(c, m)-v\left(c_{\mathrm{ref}}, m\right)\right) \\
& +\left(w(k, c, m)-w\left(k, c_{\mathrm{ref}}, m\right)\right)+\eta_{\tilde{\beta}_{m}^{k, c}} .
\end{aligned}
$$

The first three terms can be combined to

$$
\tilde{u}(k, m):=u(k, m)+v\left(c_{\mathrm{ref}}, m\right)+w\left(k, c_{\mathrm{ref}}, m\right),
$$

which is of dimension $K M$. As a separate determination of $\tilde{u}(k, m), v(c, m)$ and $w(k, c, m)$ from $\hat{\tilde{\beta}}_{m}^{k, c}$ is not feasible due to rank-deficiency and as the integer property of $w(k, c, m)$ shall be exploited, we select a satellite $k$ that is visible both at cluster $c$ and cluster $c_{\text {ref }}$ as dual-cluster reference satellite (being denoted by $\overline{\text { ref }}$ ), and map the differential ambiguity $w(\overline{\text { ref }}, c, m)-$ $w\left(\overline{\mathrm{ref}}, c_{\mathrm{ref}}, m\right)$ to $v(c, m)$. Thus, the satellite phase bias estimate is expressed in terms of the reduced parameter set:

$$
\hat{\tilde{\beta}}_{m}^{k, c}=\tilde{u}(k, m)+\tilde{v}(c, m)+\tilde{w}(k, c, m)+\eta_{\tilde{\beta}_{m}^{k, c}},
$$

with

$$
\begin{aligned}
\tilde{v}(c, m)= & v(c, m)-v\left(c_{\mathrm{ref}}, m\right) \\
& +(w \overline{\mathrm{ref}}, c, m)-w\left(\overline{\mathrm{ref}}, c_{\mathrm{ref}}, m\right) \quad \forall c \neq c_{\mathrm{ref}}, m \\
\tilde{w}(k, c, m)= & \left(w(k, c, m)-w\left(k, c_{\mathrm{ref}}, m\right)\right) \\
& -\left(w(\overline{\mathrm{ref}}, c, m)-w\left(\overline{\operatorname{ref}}, c_{\mathrm{ref}}, m\right)\right) \quad \forall k \neq \overline{\mathrm{ref}}, c \neq c_{\mathrm{ref}}, m
\end{aligned}
$$

The meaning, dimensions and notation of the reduced parameter set are summarized in the following list.

- satellite phase biases $\tilde{u}(k, m)(\operatorname{dim} . K M)$ including

- projected satellite code biases

- projected receiver phase and code biases of reference receiver at reference cluster 
- integer ambiguity of reference receiver at reference cluster

- receiver phase biases $\tilde{v}(c, m)$ of reference receiver $(\operatorname{dim} .(C-1) M)$ including

- projected receiver phase bias of reference receiver at reference cluster

- projected integer ambiguities of reference receiver at cluster and reference cluster

- double difference integer ambiguities $\tilde{w}(k, c, m)(\operatorname{dim} . K C M-C M-K M+M)$ between reference stations at reference cluster and any other cluster

We derive the multi-cluster solution of satellite positions, clock offsets and phase biases from the single cluster solutions by stacking the estimates of all clusters in a column vector:

$$
\begin{aligned}
z=( & \left(\hat{\vec{x}}^{1,1}\right)^{\mathrm{T}}, \ldots,\left(\hat{\vec{x}}^{K, 1}\right)^{\mathrm{T}}, \ldots,\left(\hat{\vec{x}}^{1, C}\right)^{\mathrm{T}}, \ldots,\left(\hat{\vec{x}}^{K, C}\right)^{\mathrm{T}}, \\
& \delta \hat{\tilde{\tau}}^{1,1}, \ldots, \delta \hat{\tilde{\tau}}^{K, 1}, \ldots, \delta \hat{\tilde{\tau}}^{1, C}, \ldots, \delta \hat{\tilde{\tau}}^{K, C} \\
& \hat{\tilde{\beta}}_{1}^{1,1}, \ldots, \hat{\tilde{\beta}}_{1}^{K, 1}, \ldots, \hat{\tilde{\beta}}_{M}^{1,1}, \ldots, \hat{\tilde{\beta}}_{M}^{K, 1}, \ldots \\
& \left.\hat{\tilde{\beta}}_{1}^{1, C}, \ldots, \hat{\tilde{\beta}}_{1}^{K, C}, \ldots, \hat{\tilde{\beta}}_{M}^{1, C}, \ldots, \hat{\tilde{\beta}}_{M}^{K, C}\right)^{\mathrm{T}}
\end{aligned}
$$

The stacked estimates of all clusters are considered as measurements that are linear related to the satellite positions, clock offsets and phase biases, i.e.

$$
z=H x+\eta_{z}
$$

where the mapping matrix $H$ and the state vector $x$ can be split into a part referring to real-valued states and a part referring to integer-valued states:

$$
H=\left(H_{\text {real }}, H_{\text {int }}\right)
$$

and the combined real- and integer-valued states

$$
x=\left(\begin{array}{c}
x_{\text {real }} \\
x_{\text {int }}
\end{array}\right),
$$

where $x_{\text {real }}$ and $x_{\text {int }}$ are defined as

$$
\begin{aligned}
x_{\text {real }}= & \left(\left(\vec{x}^{1}\right)^{\mathrm{T}}, \ldots,\left(\vec{x}^{K}\right)^{\mathrm{T}},\right. \\
& \delta \tau^{1}-\delta \tau^{\mathrm{ref}, c_{\mathrm{ref}}}, \ldots, \delta \tau^{K}-\delta \tau^{\mathrm{ref}, c_{\mathrm{ref}}}, \\
& \delta \tau^{\mathrm{ref}, 1}-\delta \tau^{\mathrm{ref}, c_{\mathrm{ref}}}, \ldots, \delta \tau^{\mathrm{ref}, C}-\delta \tau^{\mathrm{ref}, c_{\mathrm{ref}}}, \\
& \tilde{u}(1,1), \ldots, \tilde{u}(K, 1), \ldots, \tilde{u}(1, M), \ldots, \tilde{u}(K, M), \\
& \tilde{v}(1,1), \ldots, \tilde{v}(C, 1), \ldots, \tilde{v}(1, M), \ldots, \tilde{v}(C, M))^{\mathrm{T}},
\end{aligned}
$$

and

$$
\begin{aligned}
x_{\mathrm{int}}= & (\tilde{w}(1,1,1), \ldots, \tilde{w}(K, 1,1), \ldots \\
& \tilde{w}(1, C, 1), \ldots, \tilde{w}(K, C, 1), \ldots, \\
& \tilde{w}(1,1, M), \ldots, \tilde{w}(K, 1, M), \ldots \\
& \tilde{w}(1, C, M), \ldots, \tilde{w}(K, C, M))^{\mathrm{T}} .
\end{aligned}
$$

The least-squares solution of $x$ is given by

$$
\hat{x}=\left(H^{\mathrm{T}} \Sigma_{z}^{-1} H\right)^{-1} H^{\mathrm{T}} \Sigma_{z}^{-1} z,
$$

with $\Sigma_{z}$ being the covariance matrix of $z$. 


\section{SIMULATION RESULTS}

In this section, the performance of the proposed method is analyzed. We simulated Galileo measurements on the frequencies E1 and E5a of the full Galileo constellation (27 satellites) for the IGS stations. The IGS stations were grouped in 16 clusters as shown in Fig. 2. The measurement noise was simulated as white Gaussian noise with a standard deviation of $2 \mathrm{~mm}$ for the carrier phase and of $20 \mathrm{~cm}$ for the pseudorange measurements. An epoch spacing of $100 \mathrm{~s}$ was chosen. The process noise of the satellite position errors, receiver and satellite clock offsets, receiver and satellite phase biases, troposheric zenith delays and ionospheric slant delays was modeled as Gaussian noise with a standard deviation of $1 \mathrm{~mm} /$ epoch.

Fig. 3 shows the convergence of the estimated satellite position and clock corrections using multiple clusters. The errors are below $3 \mathrm{~cm}$ for almost all epochs and satellites. A similar performance is achieved for the satellite phase bias estimates in Fig. 4. The latter ones are mostly below 0.1 cycles $(2 \mathrm{~cm})$.

Fig. 5 shows the benefit of the multi-cluster solution over the single cluster. The left subfigures refer to the single cluster and the right subfigures to the multi-cluster solution. Each line refers to a satellite pass. The single cluster solution has a poor accuracy for the parameters related to satellites rising at the edge of the cluster. On the contrary, the multi-cluster solution provides orbit corrections, satellite clock offsets and phase biases with an accuracy between $5 \mathrm{~mm}$ and $20 \mathrm{~mm}$ for all satellites.

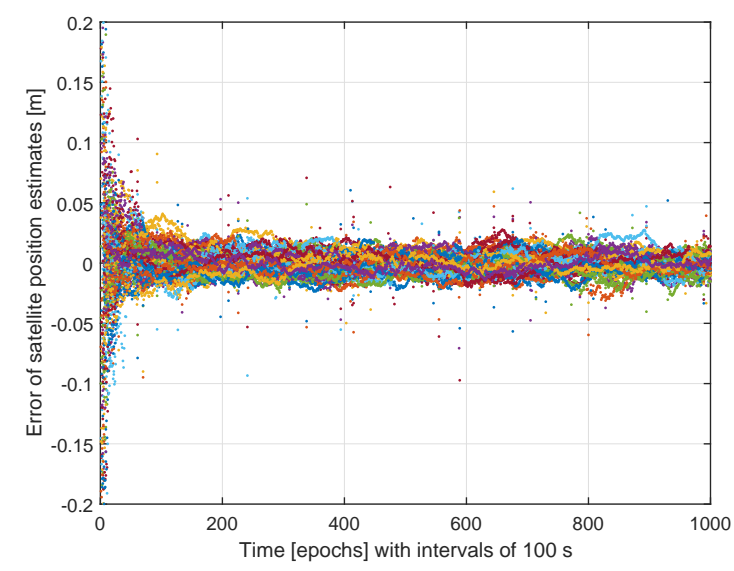

(a) Errors of satellite position estimates

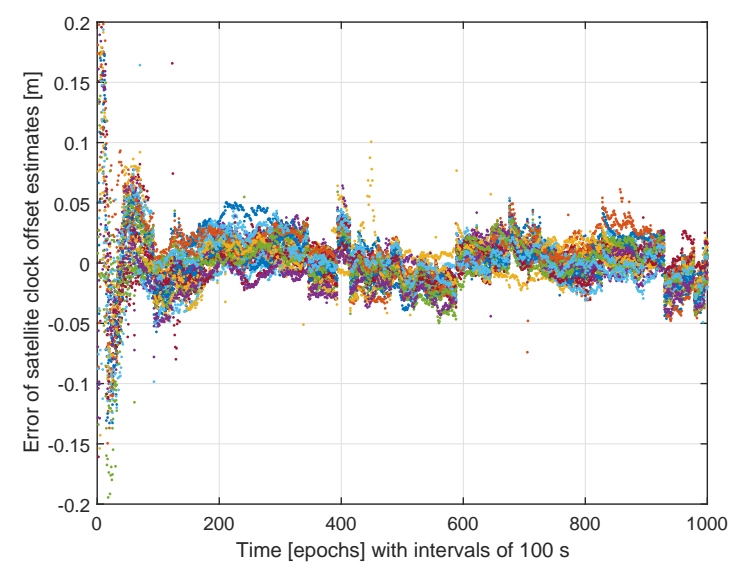

(b) Errors of satellite clock offset estimates

Fig. 3: Estimation of satellite position and clock offset corrections with multiple clusters.

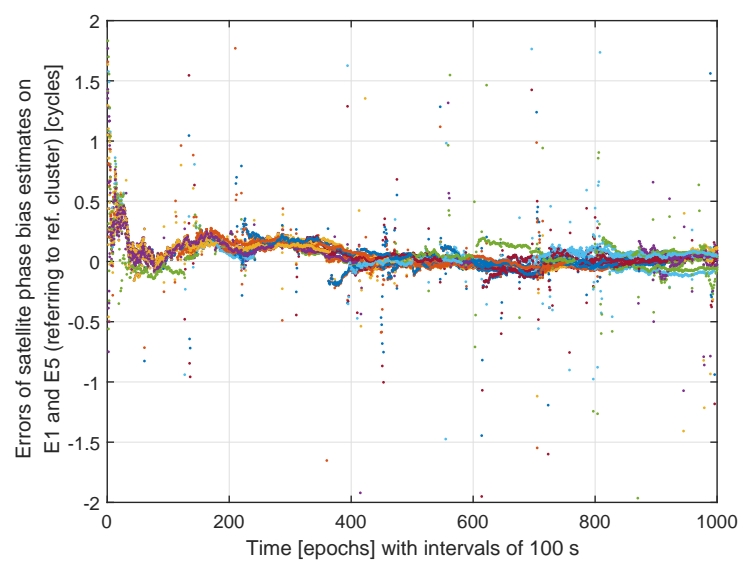

Fig. 4: Errors of satellite phase bias estimates on E1 and E5 (referring to reference cluster) in units of cycles 


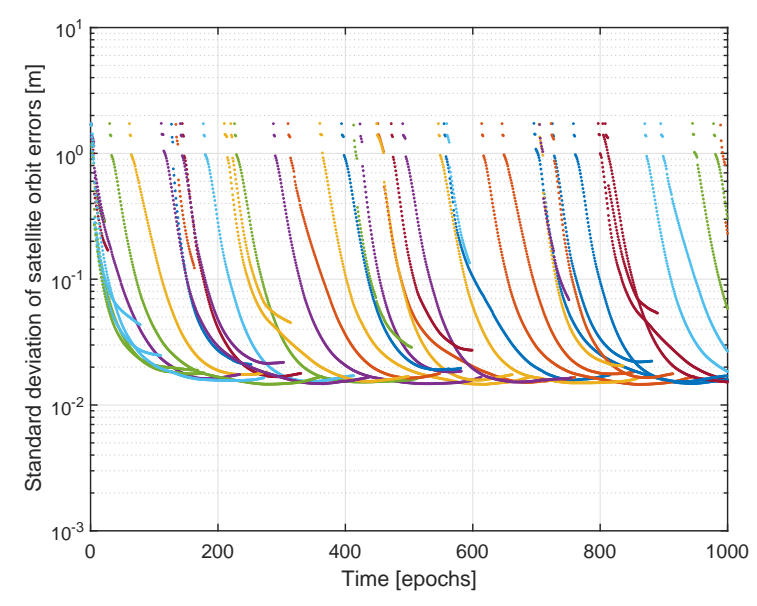

(a) Single cluster orbit corrections

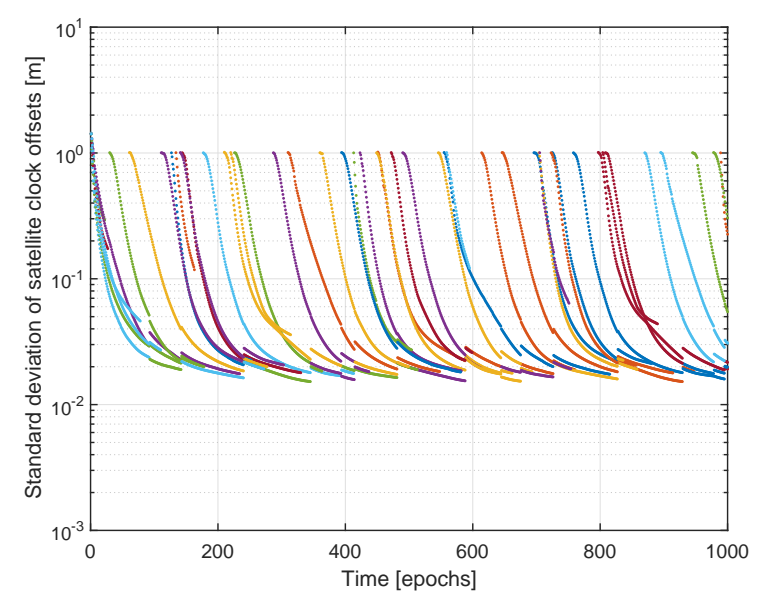

(c) Single cluster satellite clock estimation

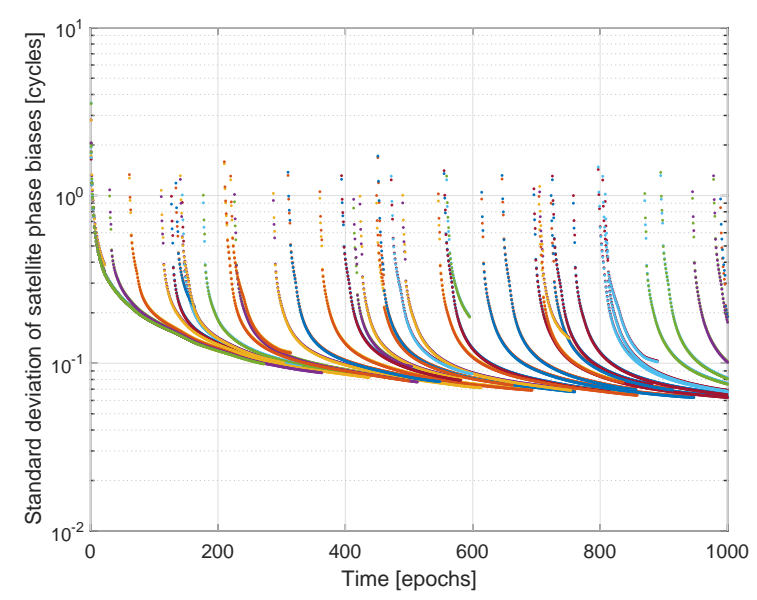

(e) Single cluster phase bias estimation

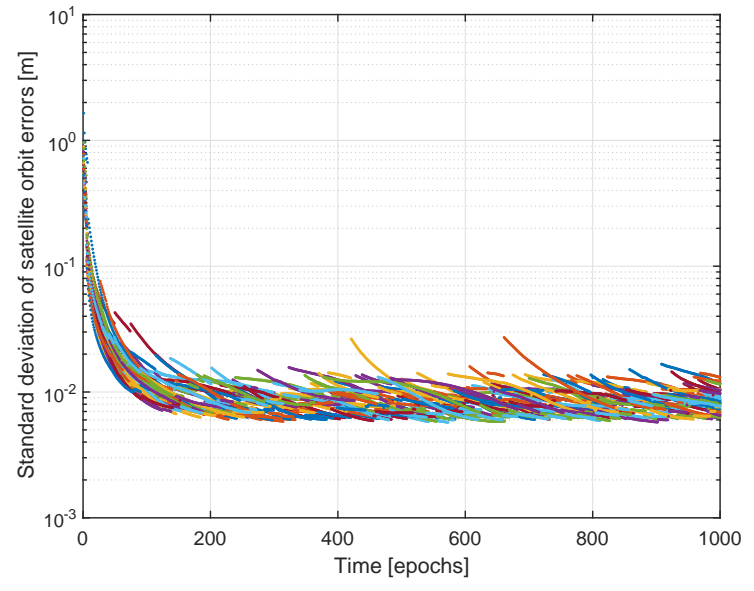

(b) Multi-cluster orbit corrections

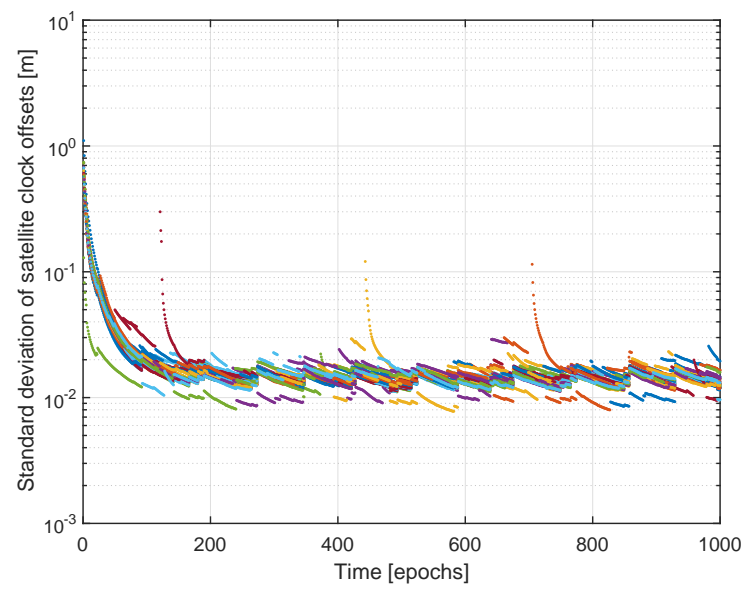

(d) Multi-cluster satellite clock estimation

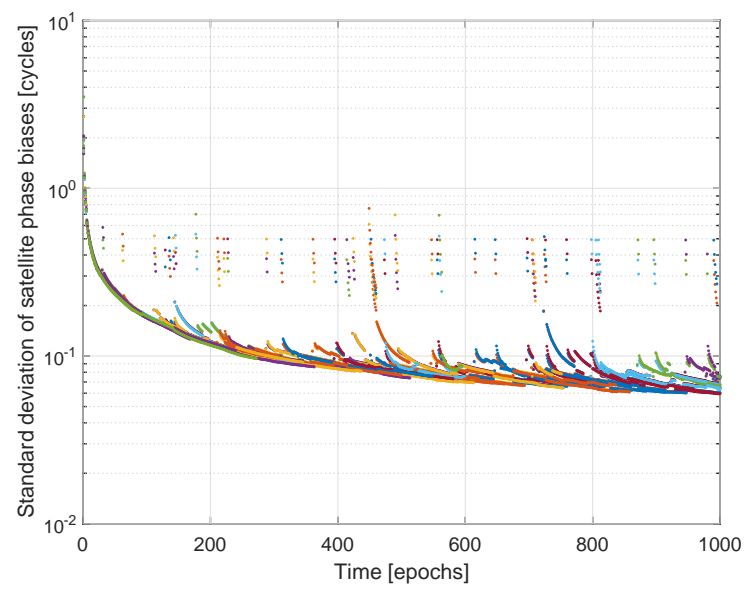

(f) Multi-cluster phase bias estimation

Fig. 5: Benefit of multi-cluster combination for estimation of orbit corrections, satellite clock offsets and satellite phase biases with fixed ambiguities. 


\section{CONCLUSION}

In this paper, a method for the estimation of orbital corrections, satellite clock offsets and phase biases was provided. The method was developed for an arbitrary number of carrier frequencies and uses undifferenced and uncombined measurements.

The method splits the global network into several clusters to reduce the dimensions of the measurements and states and, thereby, to enable integer decorrelation for ambiguity fixing. For each cluster, an individual Kalman filter was used to estimate the orbital corrections, receiver and satellite clock offsets, receiver and satellite phase biases, tropospheric zenith and ionospheric slant delays, and double difference ambiguities. This requires an optimized parameter mapping, which enables a full rank system of observation equations and fully exploits the integer property of ambiguities within each cluster and between clusters. A final least-squares adjustment combines the individual estimates of all clusters.

The method was validated with simulated Galileo measurements and showed that orbital corrections, satellite clock offsets and phase biases can be determined with an accuracy between $5 \mathrm{~mm}$ and $20 \mathrm{~mm}$ for all satellites at almost any time.

\section{REFERENCES}

[1] J.F. Zumberge, M.B. Heflin, D.C. Jefferson, M.M. Watkins, F.H. Webb, Precise Point Positioning for the efficient and robust analysis of GPS data from large networks, J. of Geophysical Research, vol. 102, pp. 5005 - 5017, 1997.

[2] J. Kouba and P. Héroux, GPS Precise Point Positioning using IGS orbit products, GPS Solutions, vol. 5, pp. 12 - $28,2000$.

[3] R. Dach, U. Hugentobler, P. Fridez and M. Meindl, Bernese GPS Software Version 5.0, User manual, Astronomical Institute, University of Bern, Switzerland, Jan. 2007.

[4] M. Ge, G. Gendt, M. Rothacher, C. Shi and J. Liu, Resolution of GPS carrier-phase ambiguities in Precise Point Positioning (PPP) with daily observations, J. of Geodesy, vol. 82, pp. 389 - 399, 2008.

[5] D. Laurichesse, F. Mercier and J.P. Berthias, Real-time PPP with undifferenced integer ambiguity resolution, experimental results, Proc. of 23rd ION GNSS, pp. 2534 - 2544, Sep. 2010.

[6] Z. Wen, P. Henkel and C. Günther, Reliable Estimation of Phase Biases of GPS Satellites with a Local Reference Network, Proc. of 53rd Int. IEEE Symp. ELMAR, Zadar, Croatia, pp. 321 - 324, Sep. 2011.

[7] P. Teunissen, The least-squares ambiguity decorrelation adjustment: a method for fast GPS integer ambiguity estimation, Journal of Geodesy, vol. 70, pp. 65 - 82, 1995.

[8] G. Blewitt, Carrier Phase Ambiguity Resolution for the Global Positioning System Applied to Geodetic Baselines up to 2000 km, J. of Geophysical Research, vol. 94, no. B8, pp. 10187 - 10203, Aug. 1989.

[9] P. Teunissen, Success probability of integer GPS ambiguity rounding and bootstrapping, J. of Geodesy, vol. 72, pp. 606 $612,1998$.

[10] A. Brack, P. Henkel and C. Günther, Sequential Best Integer-Equivariant Estimation for Geodetic Network Solutions, Proc. of ION ITM, Jan. 2013.

[11] P. Henkel, U. Mittmann and M. Iafrancesco, Real-time Kinematic Positioning with GPS and GLONASS, Proc. of 24th European Signal Processing Conference (EUSIPCO), IEEE, Budapest, Hungary, Aug. 2016. 